\title{
Geographical variation in postzygotic isolation and its genetic basis within and between two Mimulus species
}

\author{
Noland H. Martin ${ }^{1,2, *}$ and John H. Willis ${ }^{2}$ \\ ${ }^{1}$ Department of Biology, Texas State University, 601 University Drive, San Marcos, TX 78666, USA \\ ${ }^{2}$ Department of Biology, Duke University, PO Box 90338, Durham, NC 27708, USA
}

\begin{abstract}
The aim of this study is to investigate the evolution of intrinsic postzygotic isolation within and between populations of Mimulus guttatus and Mimulus nasutus. We made 17 intraspecific and interspecific crosses, across a wide geographical scale. We examined the seed germination success and pollen fertility of reciprocal $F_{1}$ and $F_{2}$ hybrids and their pure-species parents, and used biometrical genetic tests to distinguish among alternative models of inheritance. Hybrid seed inviability was sporadic in both interspecific and intraspecific crosses. For several crosses, Dobzhansky-Muller incompatibilities involving nuclear genes were implicated, while two interspecific crosses revealed evidence of cytonuclear interactions. Reduced hybrid pollen fertility was found to be greatly influenced by Dobzhansky-Muller incompatibilities in five out of six intraspecific crosses and nine out of 11 interspecific crosses. Cytonuclear incompatibilities reduced hybrid fitness in only one intraspecific and one interspecific cross. This study suggests that intrinsic postzygotic isolation is common in hybrids between these Mimulus species, yet the particular hybrid incompatibilities responsible for effecting this isolation differ among the populations tested. Hence, we conclude that they evolve and spread only at the local scale.
\end{abstract}

Keywords: speciation; reproductive isolation; hybrid sterility; hybrid inviability; asymmetric isolation; Bateson-Dobzhansky-Muller incompatibilities

\section{INTRODUCTION}

One of Darwin's fundamental evolutionary insights was his realization that the raw 'materials for natural selection to act on and accumulate' are the often subtle differences among individuals within species (Darwin 1859). He argued that the gradual accumulation of slight heritable variations by natural selection leads to substantial phenotypic evolution, which ultimately results in adaptive divergence and the origin of new species. Today, most evolutionary biologists consider that, for sexually reproducing organisms, speciation involves the evolution of reproductive isolating barriers that reduce the formation of fertile hybrids, and studies typically reveal multiple prezygotic and postzygotic barriers contributing to the total isolation between species (Chari \& Wilson 2001; Schluter 2001; Ramsey et al. 2003; Kay 2006; Nosil et al. 2006; Martin \& Willis 2007; Lowry et al. 2008; Matsubayashi \& Katakura 2009).

It is relatively easy to understand the evolution of prezygotic isolating barriers as indirect consequences of differential adaptation, divergent sexual selection or (although controversial) through reinforcement (reviewed by Coyne \& Orr 2004). However, the

\footnotetext{
* Author for correspondence (nm14@txstate.edu).
}

Electronic supplementary material is available at http://dx.doi.org/ $10.1098 /$ rstb.2010.0030 or via http://rstb.royalsocietypublishing.org.

One contribution of 18 to a Discussion Meeting Issue 'Genetics and the causes of evolution: 150 years of progress since Darwin'. evolution of intrinsic postzygotic isolation is more paradoxical. After all, as Darwin clearly stated, natural selection should not favour postzygotic isolation, since the 'sterility of hybrids could not possibly be of any advantage to them' (Darwin 1859, p. 221). For example, chromosomal rearrangements may cause partial $F_{1}$ hybrid sterility, especially in plants (White 1969; Coyne \& Orr 2004; Rieseberg \& Willis 2007), because of the production of aneuploid gametes. But selection against heterokaryotypes must also have occurred during the initial spread of novel rearrangements within species, suggesting that selection may have been overwhelmed by random genetic drift and/or minimized by homozygosity owing to high rates of inbreeding (e.g. Wright 1941; Lande 1985; Charlesworth 1992).

Instead, Darwin suggested that postzygotic barriers are probably 'not a specially acquired or endowed quality, but. . . incidental on other acquired differences' (Darwin 1859, p. 221). Decades of genetic studies have provided ample evidence that hybrid sterility and lethality are often caused by epistatic, multilocus genetic incompatibilities (Coyne \& Orr 2004) in a manner consistent with what is often termed the Dobzhansky-Muller model (Bateson 1909; Dobzhansky 1937; Muller 1940). Such Dobzhansky-Muller incompatibilities are thought to arise 'incidental' to evolutionary processes, such as genetic drift or directional selection, but a detailed understanding of the ecological and evolutionary mechanisms involved remains elusive. Examples of incompatibilities that are polymorphic within species 
Table 1. Locations of sympatric and allopatric populations of M. guttatus and M. nasutus used in the study. All populations were originally collected as seeds from wild plants.

\begin{tabular}{|c|c|c|c|c|}
\hline geographical region & $\begin{array}{l}\text { population } \\
\text { abbreviation }\end{array}$ & species & $\begin{array}{l}\text { allopatric/ } \\
\text { sympatric }\end{array}$ & location \\
\hline \multirow[t]{3}{*}{ Oregon Cascades } & IM & M. guttatus & allopatric & iron mountain, Hwy. 20, Linn Co., OR \\
\hline & CGR & M. guttatus & allopatric & Cougar Reservoir, Lane Co., OR \\
\hline & SF & M. nasutus & allopatric & Sherar's Falls, Tygh Valley, Wasco Co., OR \\
\hline \multirow[t]{4}{*}{$\begin{array}{l}\text { northern California } \\
\text { Coast Range }\end{array}$} & LG6 & M. guttatus & allopatric & $\begin{array}{l}\text { Hwy } 128 \text { and Berryessa-Knoxville Rd. jct. } \\
\text { Napa Co., CA }\end{array}$ \\
\hline & NDR2 & M. nasutus & allopatric & 2 mi. south of Dos Rios, Mendocino Co., CA \\
\hline & GMD & M. guttatus & sympatric (NMD) & Lake Berryessa, Solano Co., CA \\
\hline & NMD & M. nasutus & sympatric (GMD) & Lake Berryessa, Solano, Co., CA \\
\hline \multirow{6}{*}{$\begin{array}{l}\text { central California } \\
\text { Sierra Nevadas }\end{array}$} & GCC & M. guttatus & allopatric & Chinese Camp, Tuolumne Co., CA \\
\hline & NCL & M. nasutus & allopatric & Cherry Lake Rd. off Hwy. 120, Tuolumne Co., CA \\
\hline & MED & M. guttatus & sympatric (MEN) & Hwy. 120 \& Jacksonville Rd jct., Tuolumne Co., CA \\
\hline & MEN & M. nasutus & sympatric (MED) & Hwy. 120 \& Jacksonville Rd jct., Tuolumne Co., CA \\
\hline & GDP & M. guttatus & sympatric (NDP) & Don Pedro Vista Point, Hwy. 120, Tuolumne Co., CA \\
\hline & NDP & M. nasutus & sympatric (GDP) & Don Pedro Vista Point, Hwy. 120, Tuolumne Co., CA \\
\hline
\end{tabular}

Table 2. Results of a nested ANOVA for pollen viability and germination success. The factors 'species' and 'population' are nested within species. Significant effects are in bold.

\begin{tabular}{llclll}
\hline fitness character & source of variation & d.f. & sum of squares & $F$-ratio & $p$ \\
\hline germination rates & population (species) & 11 & 1.130 & 4.433 & $<$ \\
& species & 1 & 0.053 & 2.300 & 0.132 \\
total pollen & population (species) & 11 & $4.61 \times 10^{9}$ & 8.373 & $<\mathbf{0 . 0 0 0 1}$ \\
& species & 1 & $6.891 \times 10^{10}$ & 1376.8 & $<\mathbf{0 . 0 0 0 1}$ \\
pollen viability & population (species) & 11 & 3.735 & 9.257 & $<\mathbf{0 . 0 0 0 1}$ \\
& species & 1 & 5.429 & $\mathbf{1 4 8 . 0}$ & $<\mathbf{0 . 0 0 0 1}$ \\
\hline
\end{tabular}

have been identified in Drosophila (Reed \& Markow 2004; Brideau et al. 2006), Caenorhabditis (Seidel et al. 2008), Arabidopsis (Bomblies et al. 2007; Bikard et al. 2009) and Mimulus (several studies reviewed below), and these offer unique opportunities for investigating early stages in the evolution of postzygotic isolation, and may even elucidate why such incompatibilities initially arose.

The spread and fixation of hybrid incompatibility alleles in geographically structured species depends on the selection experienced by such factors. Therefore, studies of geographical variation in reproductive isolation provide insight into the evolutionary dynamics of hybrid incompatibilities. Strongly advantageous mutations that are adaptive throughout the species' ranges should spread quickly via migration (Whitlock 2003; Morjan \& Rieseberg 2004), while hybrid incompatibility alleles that are neutral or underdominant within species should spread at slower rates and be geographically restricted.

Here, we investigate patterns of genetic variation in intrinsic postzygotic isolation within and between two widely distributed, often sympatric, species of yellow monkeyflowers, the outcrossing, predominantly bee-pollinated Mimulus guttatus (Martin 2004) and the primarily self-pollinating $M$. nasutus (Vickery 1978). In nature, these species are largely isolated by numerous prezygotic and postzygotic barriers (e.g. Martin \& Willis 2007). Previous crossing and mapping studies have provided evidence for population variation in postzygotic isolation owing to reduced seed set, germination and two forms of hybrid sterility (e.g. Vickery 1978; Martin \& Willis 2007; Sweigart et al. 2007; Case \& Willis 2008). But because these studies involved crosses with a few inbred lines or populations of each species, the extent of isolation and its variation among populations is largely unknown. Here, we use crossing experiments involving multiple populations of $M$. guttatus and $M$. nasutus in order to evaluate the extent of geographical variation in postzygotic isolation and identify its genetic basis.

\section{EXPERIMENTAL DESIGN FOR EXAMINING GEOGRAPHICAL PATTERNS AND GENETIC BASIS OF HYBRID LETHALITY AND STERILITY} (a) Population sampling and crossing design We located sympatric and allopatric populations of $M$. guttatus and $M$. nasutus from three regions: Oregon's Cascade Mountains (and high desert regions immediately east), California's Northern Coastal Range and Central California's Sierra Nevadas (figure $1 a$ and table 1). We intended to sample one sympatric population from each geographical region, but no sympatric populations in Oregon were identified. Altogether, we collected seeds from 15-30 plants per species at four allopatric populations of M. guttatus (two from OR, and one from each CA region) and three of $M$. nasutus, as well as three 
(a)

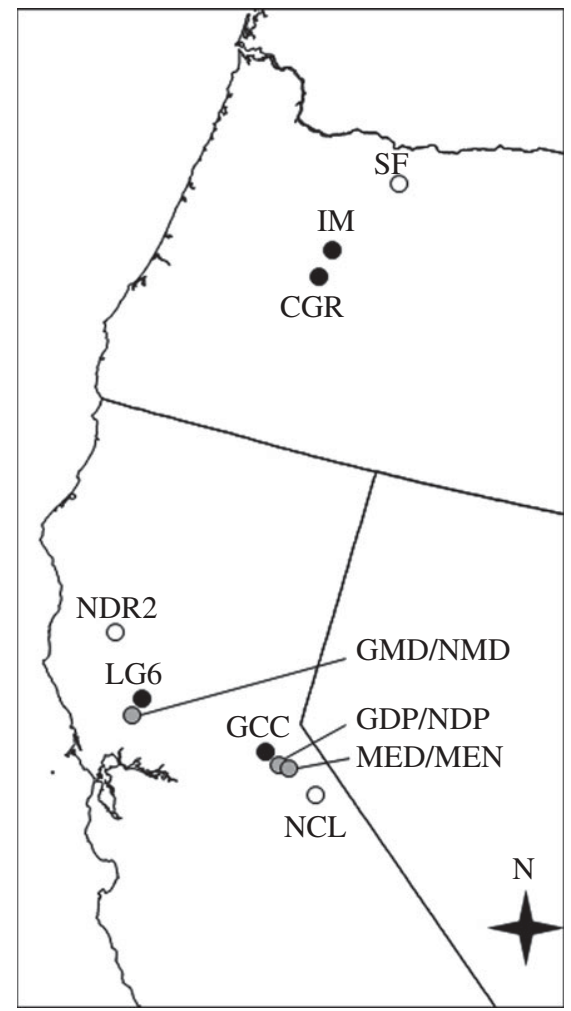

(b)

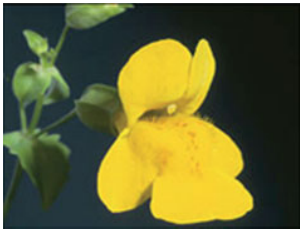

Mimulus guttatus

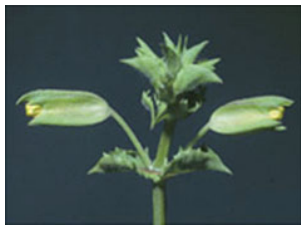

Mimulus nasutus

\begin{tabular}{|c|c|c|c|c|c|c|c|}
\hline & IM & CGR & LG6 & GCC & SF & NDR2 & NCL \\
\hline IM & $X$ & $X$ & $X$ & $X$ & X & & $X$ \\
\hline CGR & & $X$ & $X$ & $X$ & $X$ & & $X$ \\
\hline \multicolumn{2}{|l|}{ LG6 } & & $X$ & & $X$ & X & \\
\hline \multicolumn{3}{|l|}{ GCC } & & $X$ & X & & X \\
\hline \multicolumn{3}{|c|}{ SF } & & & $X$ & & $X$ \\
\hline \multicolumn{6}{|l|}{ NDR2 } & $\mathrm{X}$ & \\
\hline \multicolumn{7}{|l|}{ NCL } & $X$ \\
\hline
\end{tabular}

Figure 1. Sampling locations and crossing schematic used for this study. (a) Collection sites of M. nasutus are represented by open circles, M. guttatus by black circles, and sympatric sites by grey circles. (b) Table of allopatric crosses: cells containing an ' $\mathrm{X}$ ' indicate that reciprocal $\mathrm{F}_{1}$ and $\mathrm{F}_{2}$ hybrids were generated between the two populations (or that pure-species experimental populations were created), while a blank cell indicates that no cross was performed. (Sympatric populations were only crossed with each other.)

sympatric populations (one from the CA Coastal Range, and two from the CA Sierra Nevadas).

A total of 17 paired crosses were made between the 13 sampled populations in an incomplete diallel design (figure $1 b$ ). For each cross, 20-30 reciprocal $\mathrm{F}_{1}$ and $\mathrm{F}_{2}$ hybrids, and pure-population outbred seed families were created. For sympatric populations, species pairs were only crossed with each other. Allopatric populations were crossed to other conspecific and heterospecific allopatric populations within and among geographical regions (figure $1 b$ ).

\section{(b) Assaying intrinsic postzygotic isolation affecting seed germination and pollen fertility}

Seed germination success and pollen viability of all crosses were measured in two separate greenhouse experiments. For germination tests, seeds from 10-12 $\mathrm{F}_{1}$ fruits, and 20-24 pure-species and $\mathrm{F}_{2}$ fruits (30-125 seeds/fruit) were planted onto separate soilfilled Petri dishes (over 1800 total), randomly arranged, and the germination success of all seedlings in each dish was ascertained. 'Dish' is the unit of replication.

In a separate experiment, seeds from each seed-family created were planted $\left(20-30 \mathrm{~F}_{1}\right.$ hybrids, 50-60 $\mathrm{F}_{2}$ hybrids and pure-population parents), and a single plant was grown to flowering. The first flower of each plant was assayed for pollen viability and total pollen production using standard lactophenol-aniline blue pollen staining procedures (over 4400 flowers, detailed in the electronic supplementary material).

\section{(c) Statistical analyses}

Separate statistical analyses were conducted for each paired population cross. For all fitness characters, one-way analysis of variances (ANOVAs) were performed to determine whether there was a significant effect of 'cross type' (i.e. reciprocal $F_{1}, F_{2}$ and both parental populations) on fitness. Parental means were often significantly different from each other. Because our main goal was to detect cases of postzygotic isolation, where $F_{1}$ or $F_{2}$ hybrids perform worse than parental populations, we focus primarily on hybrid: midparent comparisons. A single planned linear contrast was also performed, comparing the mean of all four $F_{1}$ and $F_{2}$ hybrid classes with that of the average of the two parental populations.

Our experiments were designed to determine the genetic basis of postzygotic isolation. Nuclear DobzhanskyMuller incompatibilities should cause $\mathrm{F}_{2}$ fitness to be as low as or lower than $\mathrm{F}_{1}$ fitness. Alternatively, underdominant sterility factors (i.e. chromosomal rearrangements) should conform to additive-dominance model predictions, where $\mathrm{F}_{2}$ hybrid fitness is intermediate between that of $F_{1}$ hybrids and the midparent. Biometric line cross analysis (Mather 1949; Lynch \& Walsh 1998, p. 215) can be used to test whether $F_{2}$ hybrid fitness deviates significantly from additive-dominance expectations using the test statistic

$\Delta=\bar{z}\left(F_{2}\right)-\left(\frac{\bar{z}\left(P_{1}\right)+\bar{z}\left(P_{2}\right)}{4}+\frac{\bar{z}\left(F_{1}\right)}{2}\right)$ 

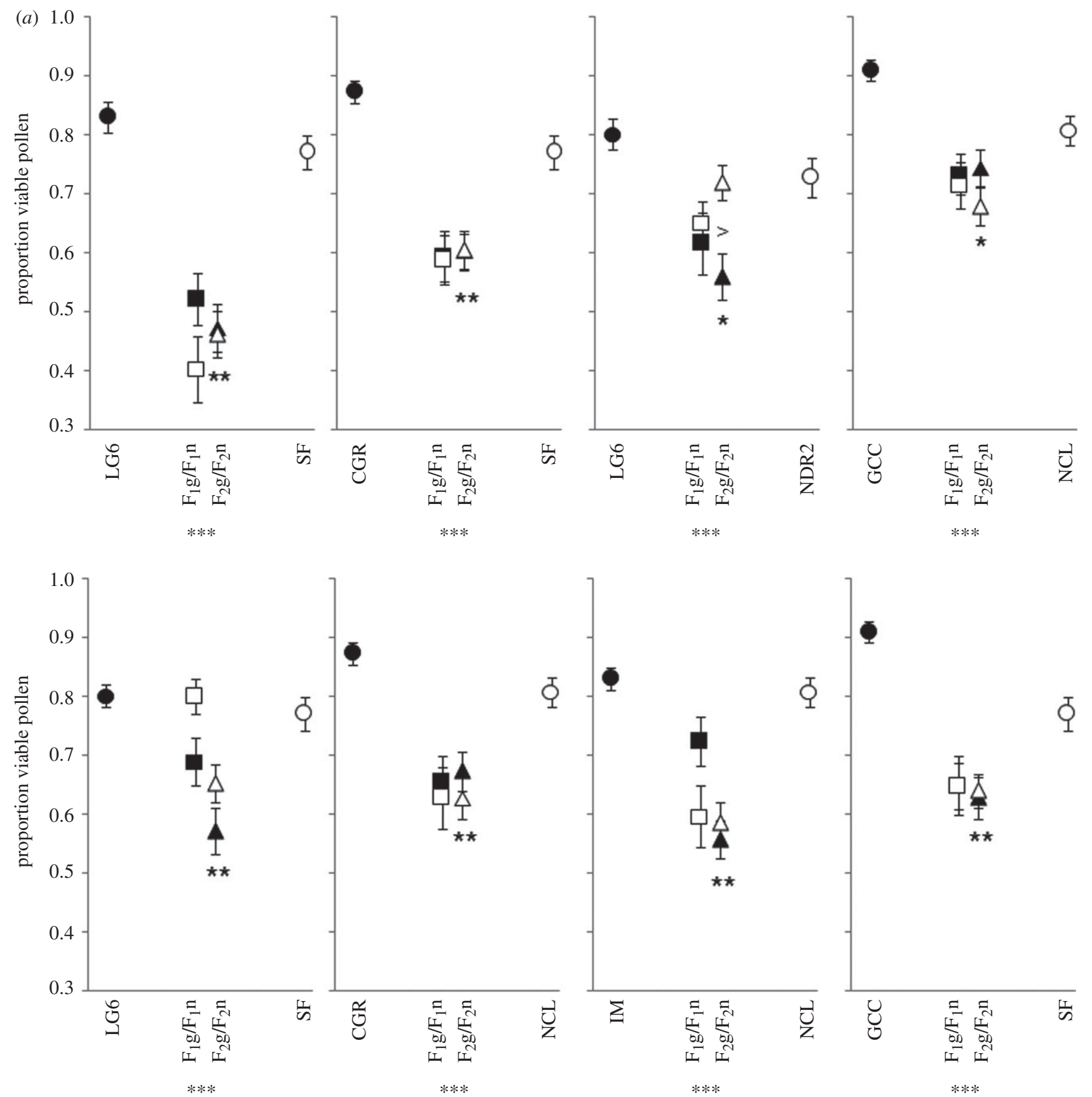

Figure 2. Germination rates for $(a)$ interspecific allopatric, $(b)$ interspecific sympatric and $(c)$ intraspecific crosses. Asterisks below the individual graphs indicate that the combined hybrid classes revealed significantly higher or lower germination rates than the midparent (linear contrasts). Asterisks above or below the $\mathrm{F}_{2}$ hybrid means indicate that the $\mathrm{F}_{2}$ hybrid class deviated significantly from additive-dominance expectations. n.s., not significant; ${ }^{*} p<0.05 ; * * p<0.01 ; * * * p<0.001$. The fill-colour of hybrids indicates that the cytoplasmic material corresponds to the parent having the same fill-colour. Circles represent pure-species populations, squares represent reciprocal $F_{1}$ populations and triangles represent $F_{2}$ populations.

where $\bar{z}$ is the mean for each class (parents $\mathrm{P}_{1}, \mathrm{P}_{2}$ and $\mathrm{F}_{1}$, $\mathrm{F}_{2}$ hybrids). The ratio $|\Delta| / \sqrt{\operatorname{Var}(\Delta)}$ provides a $t$-test for rejection of the additive-dominance model, where

$$
\begin{aligned}
\operatorname{Var}(\Delta)= & \operatorname{Var}\left[\bar{z}\left(F_{2}\right)\right]+\frac{\operatorname{Var}\left[\bar{z}\left(F_{1}\right)\right]}{4} \\
& +\frac{\operatorname{Var}\left[\bar{z}\left(P_{1}\right)\right]+\operatorname{Var}\left[\bar{z}\left(P_{2}\right)\right]}{16} .
\end{aligned}
$$

This test was used to determine the genetic basis of hybrid fitness for each cross. Because reciprocal differences in hybrid fitness were detected, tests were performed separately for each reciprocal cross.
Asymmetries were further examined using post hoc Tukey tests to determine whether reciprocal $F_{1}$ and $F_{2}$ hybrid fitnesses differed significantly.

\section{RESULTS: TESTING FOR POSTZYGOTIC ISOLATION AND ITS GENETIC BASIS}

\section{(a) Germination success}

Seed germination success for parental populations was fairly uniform and high (approx. 75-95\%), and did not differ significantly among species. Although there was significant heterogeneity among the 13 parental populations (nested ANOVA, table 2), this was largely 

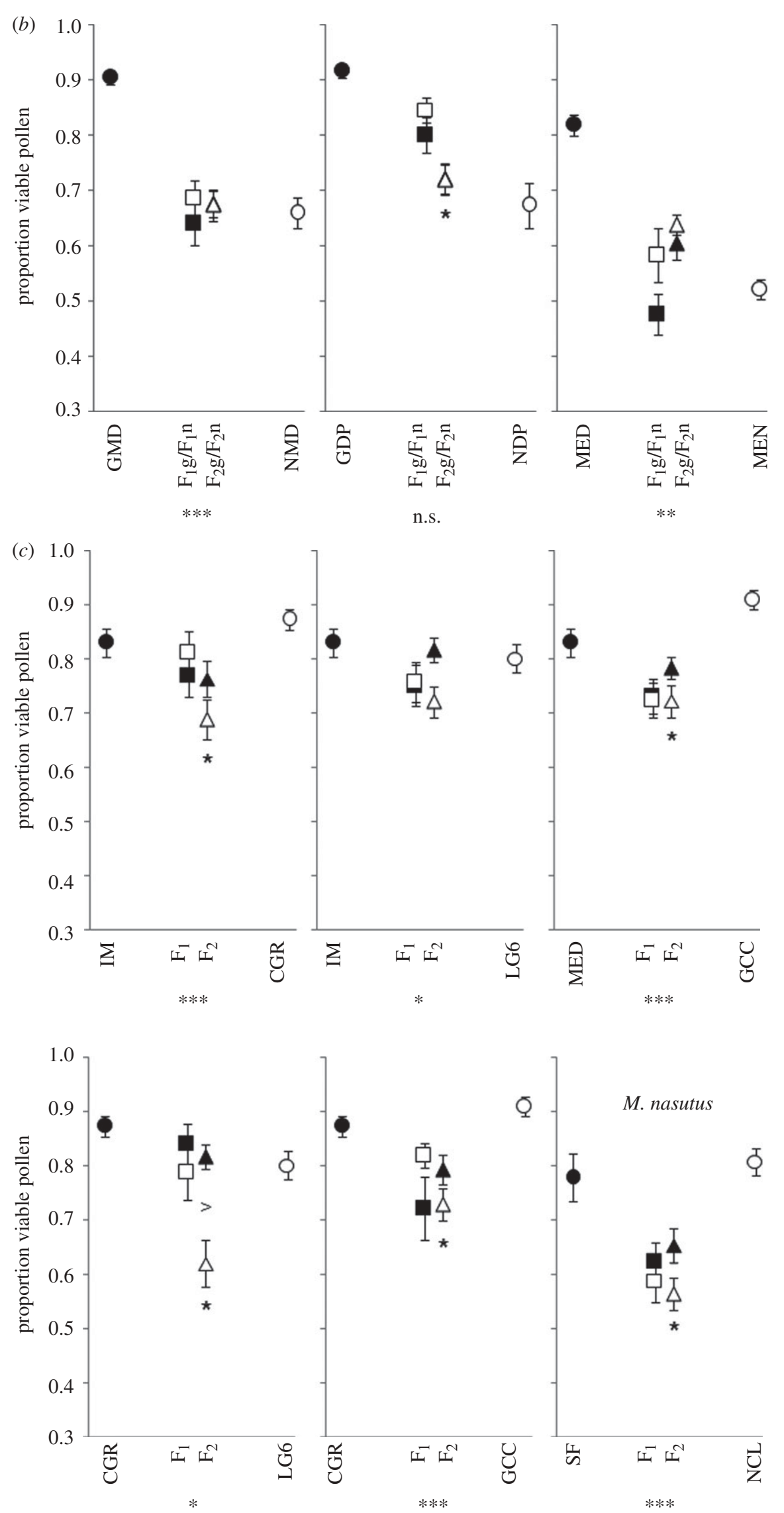

Figure 2. (Continued.)

because of the low germination success of a single $M$. nasutus population from OR (SF $\sim 55 \%$, Tukey tests).

For most crosses there was little evidence for reproductive isolation owing to hybrid seed inviability, and hybrid seed generally exhibited equal or higher germination rates than at least one of the parental progenitors, with three exceptions (Tukey tests, figure 2). In the intraspecific $M$. guttatus cross 

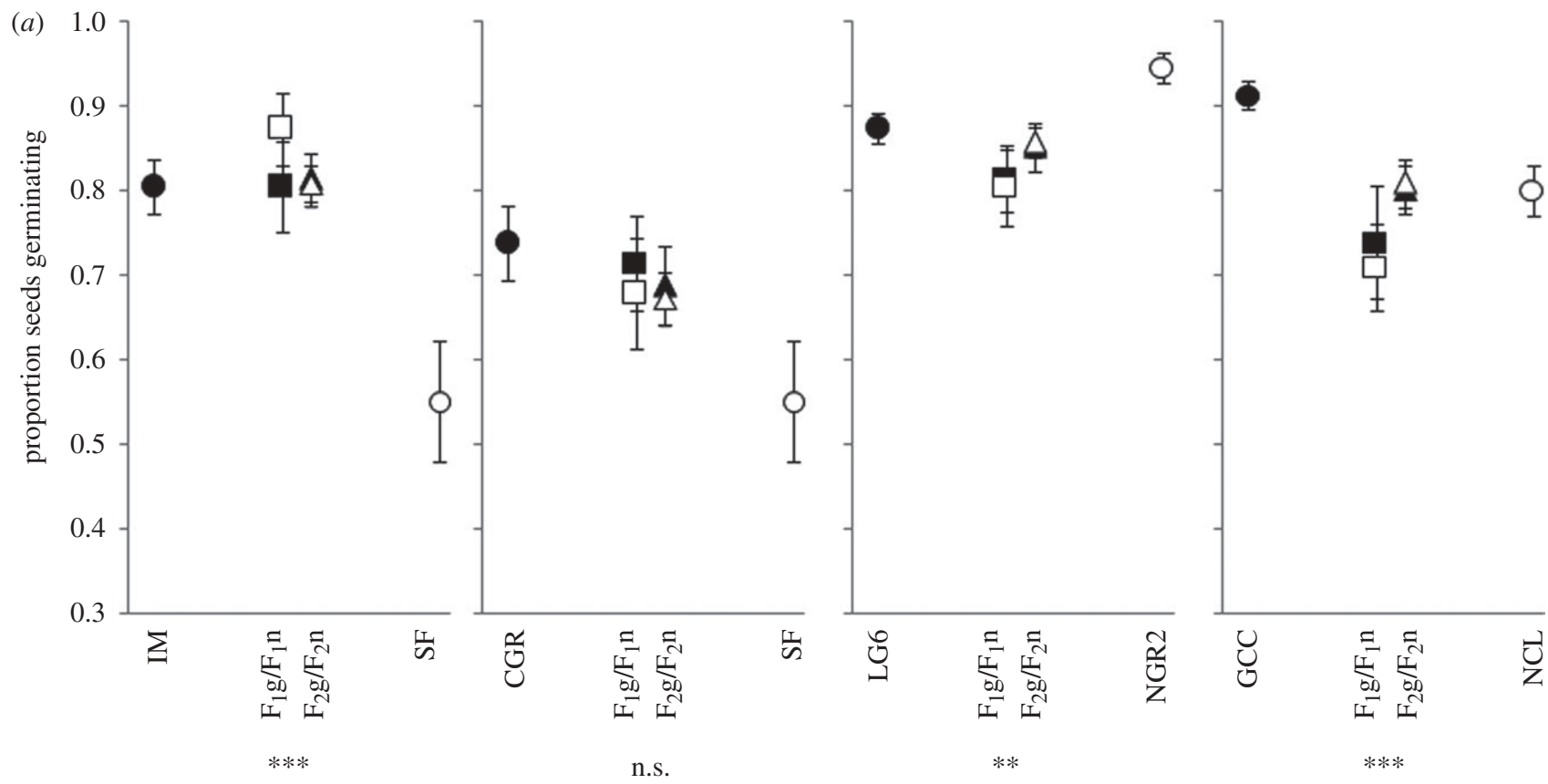

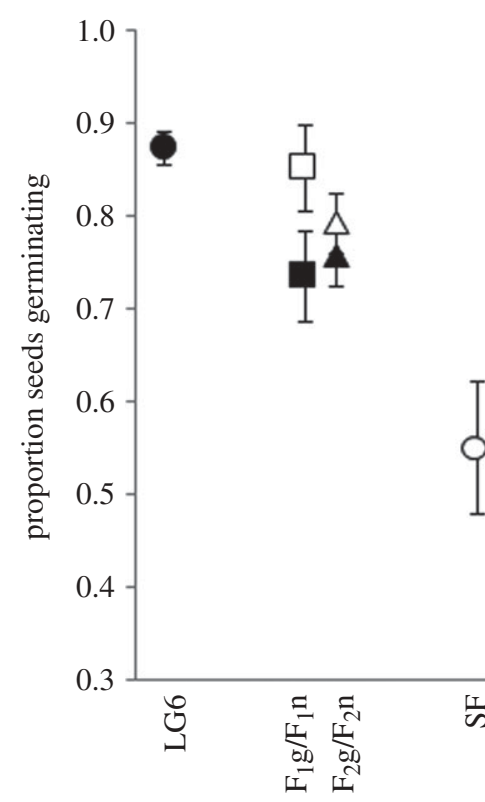

n.s.

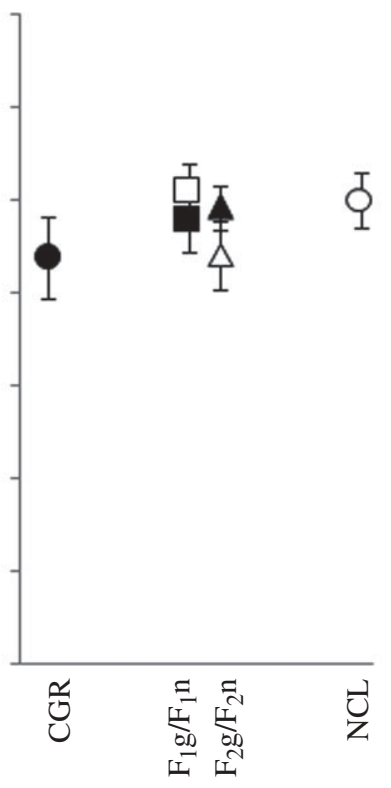

n.s.

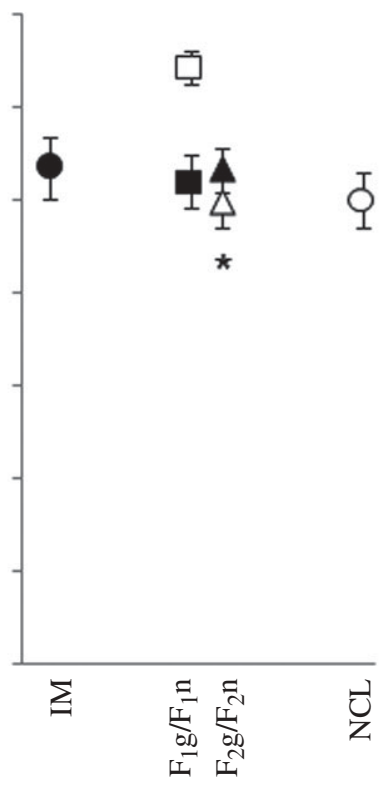

n.s.

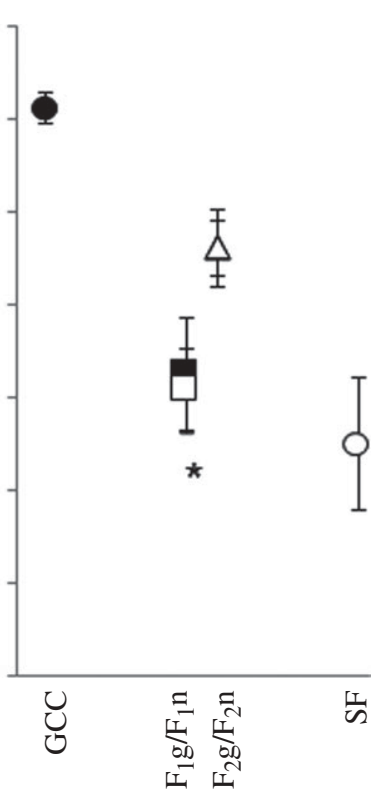

n.s.

Figure 3. Pollen viability for (a) interspecific allopatric, $(b)$ interspecific sympatric and $(c)$ intraspecific crosses. Asterisks below the individual graphs indicate that the combined hybrid classes revealed significantly higher or lower pollen viability than the midparent (linear contrasts). Asterisks above or below the $\mathrm{F}_{2}$ hybrid means indicate that the $\mathrm{F}_{2}$ hybrid class deviated significantly from additive-dominant expectations. n.s., not significant; $* p<0.05 ; * * p<0.01 ; * * * p<0.001$. The fill-colour of hybrids indicates that the cytoplasmic material corresponds to the parent having the same fill-colour. Circles represent pure-species populations, squares represent reciprocal $F_{1}$ populations and triangles represent $\mathrm{F}_{2}$ populations.

CGR $\times$ GCC, and the sympatric interspecific crosses $M E D \times M E N$ and $G M D \times N M D$, the germination success of one reciprocal $F_{1}$ hybrid class was significantly lower than either parental population (Tukey tests, figure 2). Hybrid germination rates averaged over all four hybrid classes were not significantly different from the midparent for over half of the crosses (9 of 17, linear contrasts). Hybrids germinated at significantly lower rates than the midparent for five crosses, while three crosses revealed germination success higher than the midparent. No obvious geographical (or otherwise) patterns were apparent (figure 2).
The biometrical line-cross tests revealed that $F_{2}$ hybrid germination was higher than expected under an additive-dominance model for five of the 17 population crosses, while lower than expected for only two crosses (figure 2), indicating little evidence for nuclear Dobzhansky-Muller incompatibilities affecting seed germination.

\section{(b) Pollen fertility}

(i) Total pollen

M. guttatus produced over 12 times more pollen grains than $M$. nasutus (21113 2353 and 


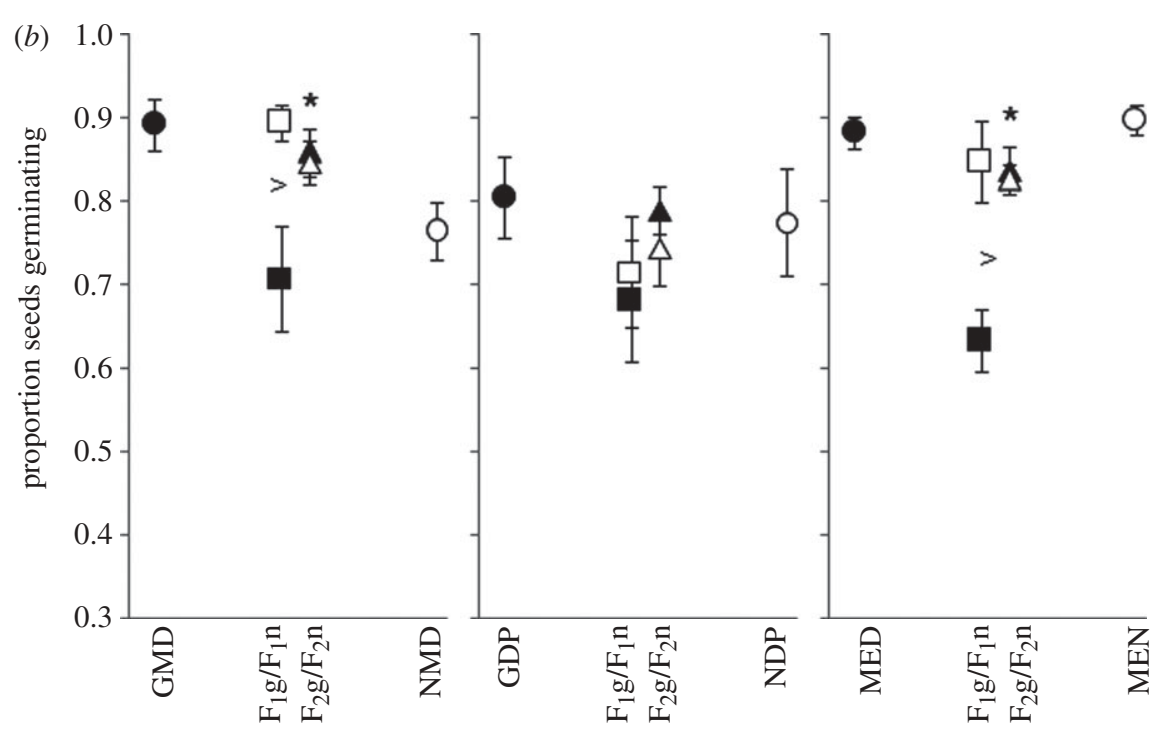

n.s.

n.s.
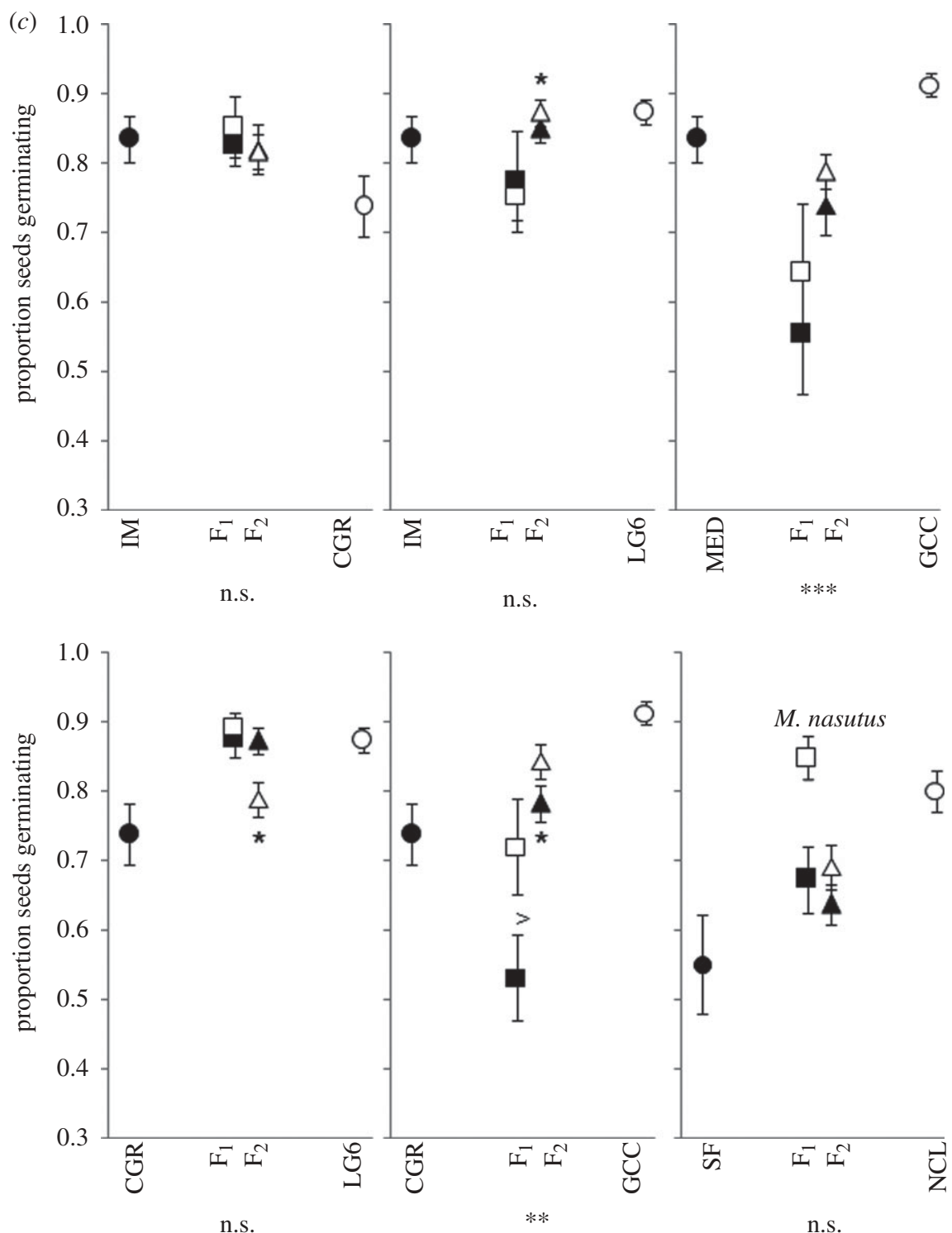

Figure 3. (Continued.)

$1639 \pm 388$, respectively, $L S M \pm 1$ s.e.), a highly significant difference (table 2 and electronic supplementary material, table S1). ANOVA revealed significant heterogeneity for total pollen production among populations of $M$. guttatus, but no significant differences among $M$. nasutus populations (table 2 and electronic supplementary material, table S1). 


\section{(ii) Pollen viability}

Mean pollen viability varied considerably among species, as well as among populations within species (ANOVA, table 2). The pollen viability of $M$. guttatus $(0.865 \pm 0.010, \quad \operatorname{LSM} \pm 1 \quad$ s.e. $)$ was significantly higher than $M$. nasutus $(0.691 \pm 0.011)$. Sympatric populations of $M$. nasutus had significantly lower pollen viability than allopatric $M$. nasutus populations $(61.4 \pm 1.8 \%, \quad 76.9 \pm 1.7 \%$, respectively, nested ANOVA: population [sympatric/allopatric], $F=37.4$, $p<0.0001)$.

For most crosses, pollen viability averaged over all four hybrid classes was consistently lower than the midparent, with reductions ranging from approximately 10 to 45 per cent (figure 3). Exceptions involved the three sympatric interspecific crosses, where the pollen fertility of $M$. nasutus populations was about as low as the four hybrid classes (post hoc Tukey tests, $p<0.05$, figure 3).

Biometrical line cross tests revealed that 14 of 17 crosses deviated significantly from an additive-dominance mode of inheritance. In every case, deviations resulted from $F_{2}$ hybrids having lower pollen viability than expected, consistent with Dobzhansky-Muller incompatibilities. Two of the three non-significant crosses involved sympatric populations with the poorly performing $M$. nasutus parental populations, making interpretations difficult. The third exception involved the intraspecific $M$. guttatus cross IM $\times$ LG6, which displayed little reduction in hybrid pollen viability.

Only two crosses exhibited significant reciprocal differences in hybrid pollen viability, and both involved $\mathrm{F}_{2}$ hybrids that inherited the CA Coast Range M. guttatus LG6 cytoplasm (figure 3). In the crosses LG6 $\times$ NDR2 and LG6 $\times$ CGR, $F_{2}$ hybrids with the LG6 cytoplasm had significantly lower pollen viability than reciprocal $\mathrm{F}_{2} \mathrm{~s}$.

\section{DISCUSSION}

Since Darwin, researchers have made tremendous progress towards understanding how ecological, genetic and evolutionary factors acting within species lead to the evolution of reproductive isolation and ultimately the origin of new species (Coyne \& Orr 2004). One problem that remains largely unresolved concerns the evolution of intrinsic postzygotic isolation. Investigating early stages in the evolution of postzygotic isolation in species, where alleles underlying postzygotic isolation are still polymorphic, is one promising approach.

Previous studies of postzygotic isolation between M. guttatus and M. nasutus have found reduced seed germination and male infertility in hybrids (Vickery 1956, 1973, 1978; Fishman \& Willis 2001, 2006; Martin \& Willis 2007). Vickery (1956, 1973, 1978) and Sweigart et al. (2007) provided evidence that postzygotic isolation within and among populations of both species varied geographically, though biometrical line crosses were not used to determine the genetic basis. In one particular cross of two inbred lines, the main nuclear and mitochondrial loci underlying two forms of hybrid male sterility were mapped, and at least some of the alleles were shown to have restricted geographical distributions (Sweigart et al. 2007; Case \& Willis 2008). However, the extent to which this isolation varied across the geographical ranges of these largely sympatric species was unclear. Here, we sought to determine the degree to which intrinsic postzygotic isolation for seed germination and male fertility varies among $M$. guttatus and $M$. nasutus populations sampled across the species' ranges, as well as to infer the genetic basis of postzygotic barriers identified. While our study only included a tiny subset of populations found in nature, several clear and interesting patterns emerged.

Only a small proportion of our crosses had reduced hybrid seed germination (and if detected, typically small), suggesting that hybrid seed inviability does not commonly arise among these species. The most striking instances of reduced hybrid seed germination involved the two sympatric crosses from the Sierra Nevada foothills of California. These crosses showed strong asymmetry, with $\mathrm{F}_{1}$ seed containing $M$. guttatus cytoplasm having approximately 25 per cent reductions in germination, whereas germination of the reciprocal $\mathrm{F}_{1} \mathrm{~s}$ was normal, suggesting a nuclear-cytoplasmic incompatibility. Since $\mathrm{F}_{2}$ seed with $M$. guttatus cytoplasm have nearly normal germination, this suggests that it may not involve simple interactions between genes in the nuclear and organelle genomes, but instead other maternal genetic or triploid endosperm effects. Either way, this incompatibility is extremely localized and is not detected in even nearby allopatric populations.

In contrast, partial hybrid male sterility was observed in almost every cross and, remarkably, is about as common in intraspecific crosses as in interspecific crosses. In the vast majority of crosses, the pattern of inheritance was consistent with the Dobzhansky-Muller model. There was no compelling evidence that chromosomal rearrangements contributed to hybrid sterility in any cross: pollen viability of $\mathrm{F}_{2} \mathrm{~s}$ generally was as low at $\mathrm{F}_{1} \mathrm{~s}$ (i.e. no rebound in fertility was observed). While we cannot rule out that rearrangements might make small contributions to hybrid sterility, these results are highly suggestive of epistatic Dobzhansky-Muller incompatibilities being the primary cause of sterility.

Deleterious nuclear-mitochondrial interactions in the form of cytoplasmic male sterility (CMS) have previously been found to cause hybrid male sterility in one interspecific Mimulus cross (IM $\times$ SF, Fishman \& Willis 2006), but were uncommon here. In that experiment, anther development was arrested in 25 per cent of the $\mathrm{F}_{2} \mathrm{~s}$ in one reciprocal cross. Such incompatibilities would not be apparent in the current assays. However, we also estimated total pollen per flower when measuring pollen viability, and thus we could inspect raw pollen-number data for asymmetrical pollen counts, and identify $\mathrm{F}_{2} \mathrm{~s}$ that lacked pollen grains. In the same $\mathrm{IM} \times \mathrm{SF}$ cross, we observed anther sterile $\mathrm{F}_{2} \mathrm{~s}$ in the expected direction. Apart from this, we found compelling evidence for this type of CMS in only one other interspecific cross $(\mathrm{LG} 6 \times \mathrm{SF})$. Again, $\mathrm{F}_{2}$ anther sterility occurred in plants with $M$. guttatus cytoplasm. Interestingly, it involves a California population of M. guttatus found by Case \& Willis (2008) to have 
another mitochondrial genome arrangement in the same region as the IM CMS mutation, suggesting that it has a novel CMS mutation (unique to LG6).

Our most striking finding is the partial hybrid male sterility of nearly every cross. This suggests that the nuclear incompatibility alleles involved are not polymorphic within species, but several considerations argue strongly against this. First, genetic mapping studies and laborious testcross analysis of the two major loci, hms1 and hms2, underlying hybrid sterility in the Oregon $\mathrm{IM} \times \mathrm{SF}$ cross revealed that, while the $M$. nasutus $h m s 2$ incompatibility allele appears fixed within that species, the M. guttatus $h m s 1$ incompatibility allele is geographically localized at intermediate frequencies only in the IM population (Sweigart et al. 2007). At least some of the individuals that we sampled from that population probably lacked that allele, since we did not observe complete sterility in any $\mathrm{F}_{2} \mathrm{~s}$ in the cross with the M. nasutus SF population (Sweigart et al. 2006). This strongly suggests that other loci are also involved in the sterility in this cross. Furthermore, the fact that M. guttatus populations previously shown to lack this incompatibility allele nonetheless produce partially sterile hybrids in intra- and interspecific crosses implies the existence of other polymorphic hybrid sterility factors.

The second line of evidence for polymorphic factors contributing to hybrid sterility comes from patterns of sterility. First, there is substantial heterogeneity in hybrid sterility among crosses. Some interspecific crosses show severe $\mathrm{F}_{2}$ hybrid breakdown (i.e. LG6 $\times S F$ ), while in many other crosses, $F_{1}$ and $F_{2}$ pollen fertility are equal. Further, the near-universal hybrid sterility cannot be explained by a single set of incompatibility factors. For example, there are several instances where a particular $M$. nasutus population is crossed to two or more $M$. guttatus populations, and hybrid sterility is observed in each cross. On its own, this might suggest that $M$. guttatus is fixed for a set of alleles that are incompatible with $M$. nasutus, and vice versa. However, this cannot explain the fact that M. guttatus populations often generate sterile hybrids when crossed with each other. Further, different populations of $M$. nasutus must have different alleles causing intraspecific hybrid sterility, as seen in our cross between Oregon and California, a possibility suggested by Sweigart et al. (2007). The only convincing explanation for these patterns is that there are many polymorphic epistatic hybrid incompatibility alleles in each species. Different populations must contain unique combinations of these alleles such that there is high fertility within populations but partial hybrid sterility in most interpopulation or interspecific crosses.

\section{CONCLUSIONS AND FUTURE DIRECTIONS}

What can these findings of highly polymorphic, epistatic hybrid incompatibilities in these species tell us about the factors that promote the evolution of intrinsic postzygotic isolation? While this study is an important first step for understanding the origin and spread of postzygotic isolation, clearly much more work is needed to identify the loci contributing to these incompatibilities. Considerable effort will be needed to elucidate the geographical distribution of the causal alleles at each locus, and to answer questions such as: what fraction of the alleles have localized distributions, like $h m s 1$, or are fixed within one species or the other, like hms2 (Sweigart et al. 2007)? It is clear from our results that some hybrid incompatibility alleles must be highly polymorphic and localized. Perhaps these alleles are selectively neutral, or at least not favoured throughout the species, or have only recently arisen and have not yet had time to spread via migration and selection among populations. The recent development of genomic resources for Mimulus (Wu et al. 2008) provides encouragement that the allelic variants contributing to the incompatibilities identified here will be further explored at the molecular and ecological level, allowing deeper insight into these earliest stages of speciation.

We thank M. Rausher, W. Morris, R. Vilgalys, L. Fishman, B. Charlesworth and A. Sweigart for comments on an early draft of this manuscript, an anonymous reviewer for critiques of later versions, S. Taylor for assistance with manuscript preparation, Duke University Greenhouse staff for plant care, and many Duke University undergraduate researchers. This work was funded through grants from the National Science Foundation to N.H.M. (DIG: DEB0073339) and J.H.W. (FIBR: EF-0328636).

\section{REFERENCES}

Bateson, W. 1909 Heredity and variation in modern lights. In Darwin and modern science (ed. A. C. Steward), pp. 85-101. Cambridge, UK: Cambridge University Press.

Bikard, D., Patel, D., Le Metté, C., Giorgi, V., Camilleri, C., Bennett, M. J. \& Loudet, O. 2009 Divergent evolution of duplicate genes leads to genetic incompatibilities within A. thaliana. Science 323, 623-626. (doi:10.1126/ science.1165917)

Bomblies, K., Lempe, J., Epple, P., Warthmann, N., Lanz, C., Dangl, J. L. \& Weigel, D. 2007 Autoimmune response as a mechanism for a Dobzhansky-Muller-type incompatibility syndrome in plants. 5, e236. (doi:10. 1371/journal.pbio.0050236)

Brideau, N. J., Flores, H. A., Wang, J. \& Barbash, D. A. 2006 Two Dobzhansky-Muller genes interact to cause hybrid lethality in Drosophila. Science 314, 1292-1295. (doi:10.1126/science.1133953)

Case, A. L. \& Willis, J. H. 2008 Hybrid male sterility in Mimulus (Phrymaceae) is associated with a geographically restricted mitochondrial rearrangement. Evolution 62, 1026-1039. (doi:10.1111/j.1558-5646.2008.00360.x)

Chari, J. \& Wilson, P. 2001 Factors limiting hybridization between Penstemon spectabilis and $P$. centranthifolius. Can. F. Bot. 79, 1439-1448. (doi:10.1139/cjb-79-12-1439)

Charlesworth, B. 1992 Evolutionary rates in partially self-fertilizing species. Am. Nat. 140, 126-148. (doi:10.1086/285406)

Coyne, J. A. \& Orr, H. A. 2004 Speciation. Sunderland, MA: Sinauer Associates.

Darwin, C. 1859 On the origin of species by means of natural selection, or the preservation of favoured races in the struggle for life. London, UK: John Murray.

Dobzhansky, T. H. 1937 Genetics and the origin of species. New York, NY: Columbia University Press.

Fishman, L. \& Willis, J. H. 2001 Evidence for DobzhanskyMuller incompatibilities contributing to the sterility 
of hybrids between Mimulus guttatus and M. nasutus. Evolution 55, 1932-1942.

Fishman, L. \& Willis, J. H. 2006 A cytonuclear incompatibility causes anther sterility in Mimulus hybrids. Evolution 60, 1372-1381.

Kay, K. M. 2006 Reproductive isolation between two closely related hummingbird-pollinated neotropical gingers. Evolution 60, 538-552.

Lande, R. 1985 The fixation of chromosomal rearrangements in a subdivided population with local extinction and colonization. Heredity 54, 323-332. (doi:10.1038/ hdy.1985.43)

Lowry, D. B., Modliszewski, J. L., Wright, K. M., Wu, C. A. \& Willis, J. H. 2008 The strength and genetic basis of reproductive isolating barriers in flowering plants. Phil. Trans. $R$. Soc. B 363, 3009-3021. (doi:10.1098/rstb.2008.0064)

Lynch, M. \& Walsh, B. 1998 Genetics and analysis of quantitative traits. Sunderland, MA: Sinauer Associates.

Martin, N. H. 2004 Flower size preferences of the honeybee (Apis mellifera) foraging on Mimulus guttatus (Scrophulariaceae). Evol. Ecol. Res. 6, 777-782.

Martin, N. H. \& Willis, J. H. 2007 Ecological divergence associated with mating system causes nearly complete reproductive isolation between sympatric Mimulus species. Evolution 61, 68-82. (doi:10.1111/j.1558-5646. 2007.00006.x)

Mather, K. 1949 Biometrical genetics. London, UK: Methuen.

Matsubayashi, K. W. \& Katakura, H. 2009 Contribution of multiple isolating barriers to reproductive isolation between a pair of phytophagous ladybird beetles. Evolution 63, 2563-2580. (doi:10.1111/j.1558-5646.2009. 00738.x)

Morjan, C. L. \& Rieseberg, L. H. 2004 How species evolve collectively: implications of gene flow and selection for the spread of advantageous alleles. Mol. Ecol. 13, 1341-1356. (doi:10.1111/j.1365-294X.2004.02164.x)

Muller, H. J. 1940 Bearing of the 'Drosophila' work on systematics. In The new systematics (ed. J. Huxley), pp. 185-268. London, UK: Oxford University Press.

Nosil, P., Vines, T. H. \& Funk, D. J. 2006 Perspective: reproductive isolation caused by natural selection against immigrants using divergent environments. Evolution 59, 705-719.

Ramsey, J., Bradshaw, H. D. \& Schemske, D. W. 2003 Components of reproductive isolation between the monkeyflowers Mimulus lewisii and $M$. cardinalis (Phrymaceae). Evolution 57, 1520-1534.

Reed, L. K. \& Markow, T. A. 2004 Early events in speciation: polymorphism for hybrid male sterility in Drosophila. Proc. Natl Acad. Sci. USA 101, 9009-9012. (doi:10.1073/pnas.0403106101)

Rieseberg, L. H. \& Willis, J. H. 2007 Plant speciation. Science 317, 910-914. (doi:10.1126/science.1137729)

Schluter, D. 2001 Ecology and the origin of species. Trends Ecol. Evol. 16, 372-380. (doi:10.1016/S01695347(01)02198-X)

Seidel, H. S., Rockman, M. V. \& Kruglyak, L. 2008 Widespread genetic incompatibility in $C$. elegans maintained by balancing selection. Science 319, 589-594. (doi:10.1126/science.1151107)

Sweigart, A. L., Fishman, L. \& Willis, J. H. 2006 A simple genetic incompatibility causes hybrid male sterility in Mimulus. Genetics 172, 2465-2479. (doi:10.1534/ genetics.105.053686)

Sweigart, A. L., Mason, A. R. \& Willis, J. H. 2007 Natural variation for a hybrid incompatibility between two species of Mimulus. Evolution 60, 141-151. (doi:10.1111/j.15585646.2007.00011.x)

Vickery Jr., R. K. 1956 Data on interracial and interspecific hybridizations in the section Simiolus of the genus Mimulus (Scrophulariaceae). Utah Acad. Sci. Arts. Lett. 33, 45-64.

Vickery Jr., R. K. 1973 Crossing barriers in the yellow monkey flowers of the genus Mimulus (Scrophulariaceae). Evolution 18, 52-69. (doi:10.2307/2406419)

Vickery Jr., R. K. 1978 Case studies in the evolution of species complexes in Mimulus. Evol. Biol. 11, $405-507$.

White, M. J. D. 1969 Chromosomal rearrangements and speciation in animals. Annu. Rev. Genet. 3, 75-98. (doi:10.1146/annurev.ge.03.120169.000451)

Whitlock, M. 2003 Fixation probability and time in subdivided populations. Genetics 164, 767-779.

Wright, S. 1941 On the probability of fixation of reciprocal translocations. Am. Nat. 74, 513-522.

Wu, C. A., Lowry, D. B., Cooley, A. M., Wright, K. M., Lee, Y. W. \& Willis, J. H. 2008 Mimulus is an emerging model system for the integration of ecological and genomic studies. Heredity 100, 200-230. (doi:10.1038/ sj.hdy.6801018) 